\title{
40. Komplexe Überdachung II: Luxemburg. Die Genese einer neuen Nationalsprache
}

Der Beitrag behandelt die komplexe Sprachensituation Luxemburgs, mit Fokus auf den germanischen Varietäten, i.e. Deutsch und Luxemburgisch, im Hinblick auf Überdachung und ihre sprachgeschichtlichen Verschiebungen. In dieser varietätenlinguistischen Perspektive liegt der Schwerpunkt auf der äußeren Sprachgeschichte; Aspekte der inneren Sprachgeschichte werden aus Platzgründen lediglich anhand ausgewählter Beispiele thematisiert.

\section{Gesamtsituation der komplexen Überdachung}

Das luxemburgische Sprachgebiet war bedingt durch seine Randlage an der germanischromanischen Grenze seit jeher bedeutenden Sprachkontakten ausgesetzt, die zur Entstehung einer komplexen Mehrsprachigkeit führten. Durch die komplexe territoriale, dynastische und politische Entwicklungsgeschichte war das Gebiet häufigen Herrschaftswechseln und Gebietsveränderungen unterworfen (vgl. Pauly 2013). Bis 1839 erstreckte sich das Gebiet auch über die germanisch-romanische Sprachgrenze in das wallonische Sprachgebiet hinein (vgl. die Karten in Uhrmacher 2009 und Gilles 2009). Unmittelbar nach der Gründung des Großherzogtums Luxemburg in seinen heutigen Grenzen (1839) wurde die Zweisprachigkeit Deutsch-Französisch auch staatlich akzeptiert und fixiert. Das zweisprachige Schulsystem, eingeführt 1843, das in seinen Grundzügen bis heute unverändert ist, sollte eine weitreichende schriftliche und mündliche Sprachkompetenz in den beiden Sprachen garantieren. Wie im übrigen deutschsprachigen Gebiet wurden im überwiegend ländlich geprägten Luxemburg moselfränkische Dialekte gesprochen. In der germanistischen Dialektologie wird der luxemburgische Raum dementsprechend dem Westmoselfränkischen zugeordnet.

In der heutigen mehrsprachigen Gesellschaft nimmt das Französische als meistverwendete Schriftsprache der Institutionen und als lingua franca vieler öffentlicher Situationen eine herausragende Stellung ein, gegen die sich das Luxemburgische positionieren muss. Bedingt durch den hohen Ausländeranteil von $48 \%$ wird das Luxemburgische, obwohl Nationalsprache, nicht von allen Einwohnern beherrscht. In der letzten Volkszählung von 2011 bezeichneten 55,8 \% der Befragten das Luxemburgische als ihre Hauptsprache (Gesamteinwohnerzahl ca. 500.000). Hingegen gaben 70,5 \% an, Luxemburgisch regelmäßig zu verwenden. Daraus kann abgleitet werden, dass ca. $15 \%$ der Ausländer Luxemburgisch als Fremdsprache gelernt haben (Fehlen/Heinz 2016).

Die Analyse zur Dynamik der Überdachung wird zeigen, dass sich dialektale Varietäten soziolinguistisch und strukturlinguistisch zu einem neuen einzelsprachlichen Sprachsystem entwickeln. Zur exakteren terminologischen Trennung soll im Folgenden der Begriff 'Luxemburgisch' (lux. Lëtzebuergesch ['lətsəbuəjə]], Lëtzebuerger Sprooch ['lətsəbuəje Spro:x]) zur Bezeichnung der Einzelsprache erst für die Periode ab dem Zweiten Weltkrieg reserviert werden. Für die gesamte Zeit davor ist es angebracht, den Begriff 'moselfränkischer Dialekt auf luxemburgischem Gebiet' zu verwenden, der zur besseren Lesbarkeit auf 'Moselfränkisch' gekürzt wird. Wenn Dialekte im weiteren moselfränkischen Dialektgebiet 
thematisiert werden sollen, dann erfolgt eine entsprechende Attribuierung (z.B. 'Moselfränkisch in Deutschland').

\section{Dialektologische Einordung}

Dialektologisch betrachtet ordnet sich das Moselfränkische/Luxemburgisch in das so genannte 'Historische Westdeutsche' oder 'Rheinische' ein (zur Neufassung der traditionellen Dialekteinteilung vgl. Schmidt/Möller, Art. 16 in diesem Band). Beckers' (1980: 469) Karte des West(mittel)deutschen in Abb. 1 illustriert die Einordnung des Moselfränkischen/Luxemburgischen in das Westmitteldeutsche. Demnach ist das Gebiet in den Rheinischen Fächer eingelagert, gelegen zwischen der dat/das- (Hunsrückbarriere), der Dorf/Dorp-Linie (Eifelbarriere) und der op/of-Isoglosse (vgl. Abb. 1, Isoglossen 6, 2 und 4). Gemäß Frings' und Wredes einflussreicher Einteilung des Mittelfränkischen wurde der Luxemburger Raum dem (West-)Moselfränkischen zugerechnet. Diese Einteilung wurde von der sich langsam etablierenden luxemburgischen Sprachforschung übernommen, sodass Bruch (1953: 113) gar von der "Ix. [=luxemburgischen, PG] Linguistik im Schlepptau der rheinischen" spricht. Für Bach (1933) ist Luxemburg zwar in den "trierischen Kulturkreis eingelagert" (Bach 1933: 5), jedoch aufgrund seiner Randlage zur Romania ein klassisches Reliktgebiet mit zahlreichen Sonderentwicklungen. Auch wenn Bach von einer 'Verkehrsgemeinschaft Luxemburg' spricht, so ist für inn das gesamte Gebiet integraler Teil des Moselfränkischen: "Nur gelegentlich ist (was näher zu erforschen sein wird) innerhalb dieses Sprachraums die heutige deutsch-luxemburgische Landesgrenze auch Sprachgrenze, eine Entwicklung, die sich erst in jüngster Zeit durchgesetzt haben kann" (Bach 1933: 8). Wiesinger (1983) entwickelte eine weitere Einteilung auf Basis des Vokalismus, wobei die angesetzten neun Teilräume die extreme Zersplitterung des Moselfränkischen belegen. Dabei werden jedoch weite Teile Luxemburgs zu einem mehr oder weniger einheitlichen moselfränkischen Raum zusammengefasst. Ein weiteres Gebiet bildet der ripuarisch beeinflusste Norden Luxemburgs zusammen mit der Westeifel (vgl. auch Herrgen/Schmidt 1989).

Die umfangreichsten Studien zur sprachhistorischen und dialektologischen Einordnung des Luxemburgischen stammen von dem Luxemburger Sprachhistoriker Robert Bruch (19201959), der die regionale Variation des Raumes in einen breiten Rahmen der germanischen und romanischen Sprachgeschichte einzuordnen versucht (Bruch 1953, 1954a, 1955, 1963). Ohne den Dialektstatus je infrage zu stellen, legt Bruch die tieferen Entwicklungslinien des luxemburgischen Moselfränkischen frei, das während mehrerer Jahrhunderte durch fränkische Migrationsbewegungen im Kontakt mit romanischen Varietäten geprägt wurde. Seine These eines 'salisch-fränkischen Kreislaufs', i.e. der Mischung fränkischer Superstrate mit romanischen Substraten im 'Pariser Becken', blieb in der Rezeption nicht unwidersprochen; vgl. die Kritik an z.T. nicht belegbaren Annahmen in Schützeichel (1976). 


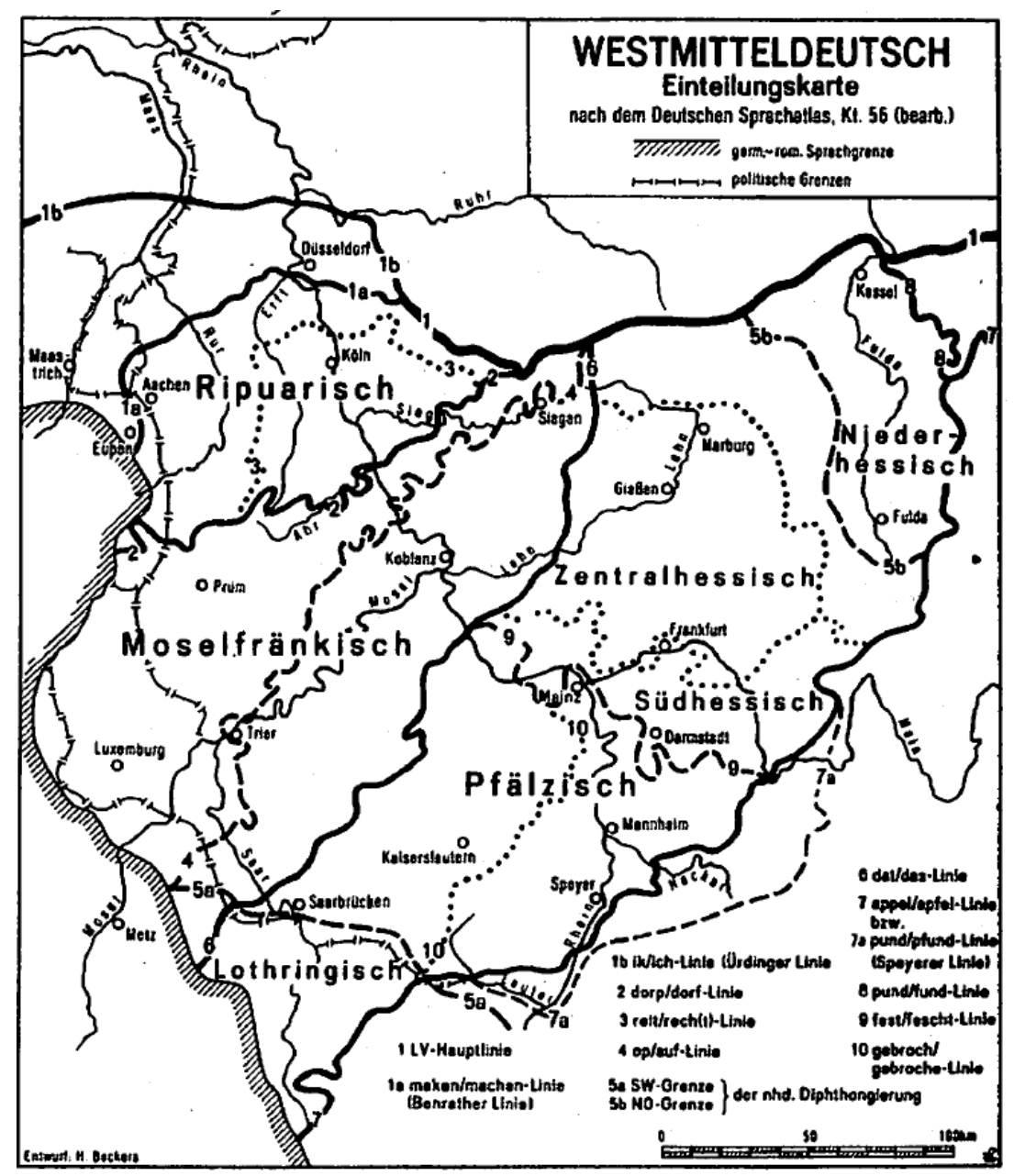

Abbildung 1 Einteilungskarte des Westmitteldeutschen aus Beckers (1980: 469)

Die dialektale Variation sowie das Verhältnis zu den Nachbardialekten wurden bereits früh erforscht. Schon 1879 wurde von John Meier die erste Erhebung von Wenkers Fragebogen durchgeführt und eine zweite Erhebung, ergänzt um phonografische Aufnahmen, erfolgte 1924/25 durch den ungarischen Sprachwissenschaftler Richard Huss im Kontext der Überprüfung der Urheimatshypothese der Siebenbürger Sachsen (Huss 1927). Ziel war es, mit vergleichbarer Methode an die kartografische Erschließung des deutschen Sprachraums anzuschließen (vgl. Gilles/Moulin 2008) sowie die interne regionale Variation zu erfassen (vgl. die Ortsgrammatiken von Hardt 1843, Klein 1855, Engelmann 1910, Palgen 1931, 1948, 1954, Bruch 1952). Auf der Basis des Luxemburgischen Sprachatlasses (LSA) (1963) kann eine interne Aufgliederung in fünf Dialektregionen (Norden, Osten, Süden, Westen, Zentrum) angesetzt werden, die sich in unterschiedlichen Dialektalitätsgraden voneinander unterscheiden (vgl. Abb. 2). Das nördliche Dialektgebiet (Ösling, lux. 'Éislek') unterscheidet sich bis heute am stärksten von allen anderen Dialekten. Die sehr ländliche Struktur bedingt eine extreme Aufspaltung in kleinräumige Untermundarten (vgl. die verschiedenen nördlichen Varianten für das Beispielwort Nacht in Abb. 2). Die verschiedenen Formen der Velarisierungen belegen ripuarische Einflüsse (vgl. Bruch 1949). Darüber hinaus trennt die in ost-westlicher Richtung verlaufende Präteritalgrenze den Norden vom Rest des Landes (vgl. Krier 2015 sowie die Karte basierend auf Auswertungen des Wenkersatzes 34 in Fleischer/Schallert 2011: 131). Das schmale östliche Gebiet entlang der Grenzflüsse Sauer und Mosel zeigt weitgehende Ähnlichkeit mit den moselfränkischen Dialekten in Deutschland. Das südliche Dialektgebiet, konzentriert um die ehemaligen Berg- und Stahlwerke, ist durch wenige, doch perzeptiv saliente Merkmale charakterisiert. Im Zentrum des Landes befindet sich hinsichtlich Fläche 
und Einwohnerzahl das größte Dialektgebiet. Von Bruch wird darüber hinaus noch ein kleineres Dialektgebiet im Westen um das Städtchen Redange/Attert angenommen, das in der Einteilung in Abb. 2 jedoch weggelassen wurde, da es sich nur durch sehr wenige, lexikalisierte Merkmale konstituiert. Für weitere sprachgeschichtliche und dialektologische Details vgl. Keller (1961), Sturm (1988), Newton (1990, 1993), Schiltz (1997), Gilles im Druck.

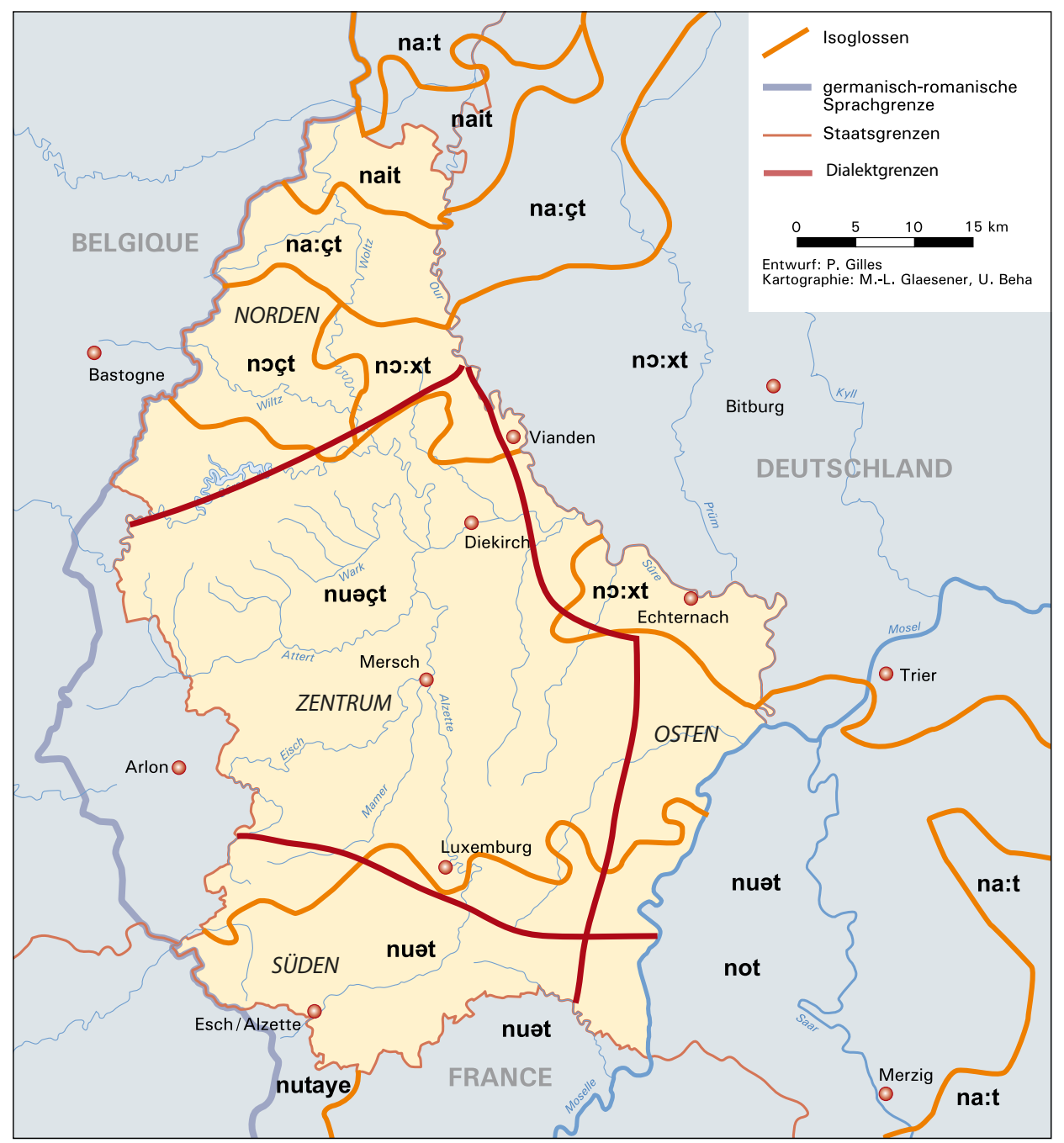

Abbildung 2 Historische, interne Dialektgliederung Luxemburgs (nach Gilles 1999), illustrative Überlagerung mit den Isoglossen des Beispielworts Nacht (basierend auf LSA 25 und DSA 361; nach Gilles 2009).

Die beschriebene Dialektsituation basiert auf Sprachdaten der ersten Hälfte des 20. Jh. und wurde umfassend im Luxemburgischen Sprachatlas (LSA) (1963) dokumentiert. Die gesellschaftlichen Modernisierungen des 20. Jh. haben auch in Luxemburg zu einem gravierenden Umbau der Dialektlandschaft geführt. Durch sozial-räumliche Mobilität wurde ein Dialektausgleich initiiert, dessen Ansätze erstmalig von Engelmann (1910) beobachtet wurde. Demnach hat sich aus den Orts- und Gebietsmundarten eine überdachende 'allgemeine luxemburgische Umgangssprache' (Koiné) entwickelt, die überregionale, standardsprachliche Funktionen übernimmt. Neben der Mehrsprachigkeit mit Deutsch und Französisch hat sich demnach aus den Orts- und Gebietsmundarten ein weiteres 'luxemburgisches' Varietätensystem herausgebildet. Über die Entstehung dieser luxemburgischen Koiné existieren kontroverse Annahmen: Nach Engelmann (1910) ist sie im Zentrum des Landes, nördlich der Hauptstadt Luxemburg-Stadt entstanden und hat sich von dort ausgebreitet. Nach Bruch (1953) soll es sich bei der Koiné jedoch um ein 
Ausgleichsprodukt handeln, an dem alle Gebietsmundarten beteiligt waren (Koinéisierung). Nach Gilles $(1999,2006)$ liegt jedoch ein vom großen zentralluxemburgischen Dialektgebiet ausgehender Dialektausgleich vor, der die Struktur der angrenzenden Gebiete nach und nach auflöst. Luxemburg-Stadt als politisches, administratives, kulturelles und infrastrukturelles Zentrum kommt dabei eine zentrale Rolle zu. Während das nördliche Dialektgebiet bis heute relativ intakt bleibt, zeigen der Süden und der Osten generationell gestaffelt deutlich stärkere Ausgleichstendenzen. Dabei ist das Vordrängen des Zentralluxemburgischen in das ostluxemburgische Gebiet hinein von besonderer Relevanz für die dialektologische Einordnung: Das ehemalige, grenzüberschreitende Dialektkontinuum mit dem Moselfränkischen in Deutschland wird durch die Staatsgrenze zerteilt, die nach und nach auch zu einer Sprachstrukturgrenze wird (Divergenz). Trotz des strukturellen Ausgleichs existieren allerdings in der laienlinguistischen Einschätzung die vier Dialektgebiete weiterhin (vgl. Neises 2013).

Wie in Deutschland hat sich also die Dialektlandschaft in Luxemburg verändert. Zwar ist das heutige Luxemburgische aus dem moselfränkischen Dialekt entstanden, doch kann heute kein gemeinsames, grenzüberschreitendes Dialektkontinuum mehr angenommen werden. Vielmehr können divergente 'Makrosynchronisierungen' (Schmidt/Herrgen 2011) beobachtet werden: Die Dialekte in Deutschland entwickeln sich unter beständigem Einfluss des Standarddeutschen in Richtung einer Regionalsprache, während in Luxemburg das Zentralluxemburgische als Ziel- und Referenzpunkt fungiert. Infolge dieser Divergenz hat sich das neu entstandene Luxemburgische aus dem Gefüge der eigentlichen deutschen Mundarten emanzipiert (Gilles 1998, 2000). Auf sprachstruktureller Ebene zeigt sich die Divergenz zu den deutschen Dialekten u.a. in folgenden, nahezu vollständigen Grammatikalisierungen: ginn 'geben'-Passiv, hatt-si-Differenzierung für weibliche Personen, 2.P.PI. als Höflichkeitsform (Dir, lech), flektierender Komplementierer (wann s de 'wenn du'), Ausbau des Allomorphs -en zur generellen Pluralmarkierung bei Verzicht auf -s-Plural, Ausbau des -s-Fugenallomorphs, Ausbau der Kompositionsfreudigkeit mit integrierten oder ad-hoc entlehnten französischen Bestandteilen etc. (Gilles im Druck).

\section{Zum Begriff der Überdachung}

Der von Kloss (1952) geprägte, spezifisch germanistische Begriff der Überdachung dient der Erfassung der hierarchischen Verhältnisse in Varietätensystemen. Dabei bildet die als Schriftsprache gebrauchte 'linguistisch zugeordnete Kultursprache', z.B. als Amts- oder Schulsprache, das Dach, von dem die überwiegend gesprochenen Mundarten funktional wie strukturell abhängig sind (Kloss 1952: 20ff.; vgl. Sinner 2013). Historisch mehrsprachige Sprachräume wie eben z.B. in Luxemburg oder in den deutschsprachigen Gebieten in Belgien lassen sich jedoch nicht (mehr) ohne Schwierigkeiten mit einem einfachen Überdachungsverhältnis erfassen. Zur Konzeptualisierung solcher komplexen Überdachungsverhältnisse wurden entsprechende Erweiterungen von Goossens (1977), Ammon (1994) sowie Darquennes (Art. 41 in diesem Band) vorgenommen. Im vorliegenden Kontext ist dabei die Differenzierung zwischen 'struktureller Überdachung' und 'funktionaler Überdachung' relevant. Während die strukturelle Überdachung der Kloss'schen Konzeption entspricht und nur für genetisch unmittelbar verwandte Sprachen zutrifft, die 'ihre' Dialekte überdacht, so kann funktionale Überdachung auch bei genetisch unverwandten Sprachen vorliegen. Dialekte können z.B. in einer Sprachgemeinschaft in informellen Kontexten verwendet werden, während hingegen eine fremde Sprache als Standardvarietät für formellere Kontexte fungiert. Durch die Einführung der funktionalen Überdachung, die von 
Kloss nicht vorgesehen war - er sprach hier von 'dachlosen Mundarten' -, können komplexe Überdachungen adäquater gefasst werden.

Kloss selbst hat die luxemburgischen Verhältnisse mehrfach thematisiert. Während er in einer frühen Publikation von einem moselfränkischen Dialekt in einem einsprachigen Land spricht (Kloss 1940), findet sich in späteren Veröffentlichungen (Kloss 1952, 1986) durchgängig die Klassifikation als Ausbaudialekt oder als Ausbausprache.

\section{4. Überdachung bis zum 2. Weltkrieg}

Die ältesten Textzeugnisse des Luxemburgischen Gebietes aus mittelhochdeutscher (Leben der Gräfin Yolanda von Vianden, vgl. Moulin 2009) und frühneuhochdeutscher Zeit (Die Rechnungsbücher der Stadt Luxemburg; vgl. Moulin/Pauly 2007) gehören im weitesten Sinne zum Moselfränkischen, weisen aber bereits in dieser Zeit partiell oberdeutsche Einflüsse auf (Ravida 2012). Da für diese Zeit noch kein standardsprachliches Bezugssystem angenommen werden kann, lässt sich auch noch keine Überdachung feststellen. Eine Diskussion der Überdachung kann sinnvollerweise erst geführt werden, wenn ein (potenzielles) Standard/Dialekt-Gefüge existiert, das sich in Luxemburg ab dem 16. Jh. herauszubilden beginnt. Indizien dafür sind z.B. die Hexenprozessprotokolle des 16./17. Jh., die in regionalsprachlichem Standarddeutsch verfasst sind, aber auch die Gründung Luxemburger Druckereien, die ab 1600 deutschsprachige Druckerzeugnisse (neben den dominanteren französischen) herstellen (Solms/Wegera 1999). Parallel zu dieser sich nach und nach verfestigenden standardsprachlichen Schriftlichkeit kann mit höchster Wahrscheinlichkeit angenommen werden, dass die, größtenteils illiterate, Bevölkerung verschiedene Dialekte des Moselfränkischen gesprochen hat. In einer der sehr spärlich überlieferten metasprachlichen Äußerungen zum Varietätengefüge aus dem Jahr 1804/05 wird die gesprochene Sprache als "un mauvais allemand, dérivant de langue teutone" bezeichnet. Weiterhin wird festgestellt, dass "le dialecte Luxembourgeois ne s'est jamais écrit; qu'il manque des mots pour exprimer autre chose, que les objets de première nécessité, et que lorsqu'on veut aller puls loin, il faut absolument recourir au haut Allemand" (zitiert nach Rinnen 1981: 11f.). Mit dieser Einschätzung ist die sich etablierende Dialekt/Standard-Konstellation adäquat erfasst.

Als das Großherzogtum Luxemburg von den Siegermächten nach den Napoleonischen Kriegen auf dem Wiener Kongress geschaffen wurde, umfasste es zunächst zwei in etwa gleich große Sprachgebiete: Im quartier wallon, gelegen im heutigen Belgien (Province du Luxembourg), wurden wallonische Dialekte gesprochen bei Überdachung durch die französische Schriftsprache. Analog dazu lag im quartier allemand das oben skizzierte Dialekt/Standard-Gefüge vor. Im Zuge der Belgischen Revolution (1830) wurde das quartier wallon abgespalten, und das übriggebliebene quartier allemand konstituiert seit 1839 das Staatsgebiet des heutigen Großherzogtums. Obwohl die Bevölkerung von dieser Zeit an als durchgehend germanophon bezeichnet werden kann, hatte sich allerdings in der Verwaltung und in den politischen Institutionen längst die Zweisprachigkeit Deutsch/Französisch etabliert, die auch in der ersten (zweisprachigen) Verfassung von 1848 in Art. 30 festgeschrieben wurde: "Der Gebrauch der deutschen und französischen Sprache steht jedem frei; es darf derselbe nicht beschränkt werden" (vgl. Fehlen 2014: 44). In der Konsequenz wurde auch im Schulsystem die Zweisprachigkeit eingeführt, in dem Deutsch als Alphabetisierungssprache und Französisch als erste Fremdsprache vorgesehen sind, ein System, dessen Grundzüge bis heute intakt sind. Bis in die zweite Jahrhunderthälfte hinein sind zwar sprachenpolitisch induzierte Verschiebungen beim Gebrauch der deutschen und französischen Schriftsprache 
zu beobachten (Beyer et al. 2014, Moliner/Ziegler 2017), doch wurde die prinzipielle Zweisprachigkeit des Landes niemals in Frage gestellt und es entstand die heutige komplexe Diglossie (vgl. Fehlen 2018).

Die gesprochenen Varietäten des Moselfränkischen werden im gesamten 19. Jh. durchgehend als 'Mundarten' oder 'Dialekte' bezeichnet, wie aus den nach und nach erscheinenden Sprachbeschreibungen hervorgeht: 'onzen Dialekt' (Meyer 1829), 'luxemburgische Mundarten' (Hardt 1843) 'unser Dialekt' (Meyer/Gloden 1845, Engelmann 1910), 'Die Luxemburger Sprache gehört, so wie die niederländische, zu den germanischen Sprachen, und zwar zu den 236 Mundarten des eigentlichen Deutschlands' (Gangler 1847), 'luxemburger deutsche Mundart' (de la Fontaine 1855), 'unsere Mundart' (Klein 1855), 'Luxemburger Mundart' (Bourg 1895, Koenig 1928), 'onst Däitsch' (de la Fontaine 1855), abwertend auch 'schlecht Däitsch' (vgl. Fehlen 2015: 69). Die pluralische Bezeichnung 'luxemburgische Mundarten' bei Hardt (1843) verweist auch darauf, dass innerhalb Luxemburgs verschiedene regionale Dialekte angenommen werden müssen. Im Gegensatz zum Moselfränkischen in Deutschland war für Luxemburg die an den niederdeutschen Raum anspielende Varietätenbezeichnung 'Platt' nie geläufig. Neben der Verwendung mit 'Luxemburg'/'luxemburgisch' ist Attribuierung mit unser(e) auffällig und verweist auf das bereits im 19. Jh. wachsende und zunächst noch verdeckte Prestige des Moselfränkischen als wichtigem (nationalen) Identifikationsfaktor. Es bestand in dieser Zeit darüber hinaus auch kein Zweifel, dass die Bezugsvarietät dieses Dialekts immer das sich etablierende Standarddeutsche ist (lux. Houdäitsch, Héi(ch)däitsch). Selbst als 1896 die erste Parlamentsrede im Dialekt gehalten wurde, gilt für den Redner C. M. Spoo: "Ons Sprôch as dě Deitsch" ('Unsere Sprache ist die deutsche') (Fehlen 2009: 25), womit er für Luxemburg ein ähnliches Varietätengefüge annimmt wie auch für andere deutsche Dialektregionen.

Ohne den Status als Dialekt in Frage zu stellen, wurde bereits im 19. Jh. der Dialekt zunehmend mit identitären Aspekten aufgeladen. Immer dann, wenn nationale Eigenschaften in den Vordergrund rückten, wurde der Dialekt als identitätsstiftende Konstruktion gebraucht. So charakterisiert Nicolas Ries in seinem 'Essai d'une psychologie du peuple luxembourgeois' von 1911 die Muttersprache der Luxemburger als 'weder Deutsch, noch Französisch' ("la langue maternelle des ses habitants, n'est ni l'allemand, ni le français, mais l'idiome luxembourgeois" (Ries 1911: 113). Die attitudinale Anbindung des Dialekts an Aspekte der Identität und der Nation verstärkt sich im Verlauf des 19. Jh. insbesondere bei der kulturellen Elite des Landes. In dieser Zeit entstehen zahlreiche nationalsymbolische Lieder (Hymnen, Feiergesänge) und es werden von Antoine Meyer, Jean-François Gangler, Edmond de la Fontaine, Michel Rodange und Michel Lentz die ersten literarischen Texte publiziert (v.a. Theaterstücke, Gedichte und das einflussreiche Versepos De Rénert (1872) von Michel Rodange). Im Gegensatz zum Moselfränkischen auf deutschem Gebiet entstand im 19. Jh. also eine erste Schriftlichkeit, die von den Autoren immer als Dialektliteratur aufgefasst wurde, die neben den großen Kultursprachen Deutsch und Französisch existiert. Newton (1996b: 183) resümiert diese Frühphase des Ablöseprozesses folgendermaßen: "All these events served further to establish Luxembourg as separate in identity from either Germany and France, and to establish Lëtzebuergesch as the hallmark of that separateness."; vgl. auch Solms/Hoffmann (1995).

Der Dialekt erfährt eine erste wichtige institutionelle Anerkennung im Schulgesetz von 1912 durch die Einführung als Schulfach, allerdings nicht als Sprachfach, sondern in Kombination mit der Landeskunde und in einem sehr geringen zeitlichen Umfang. Im Gefolge des Schulgesetzes entsteht das erste Lesebuch für die Volksschule (Welter 1914). Alphabetisierungsprache und wichtigste Schulsprache bleibt jedoch weiterhin das 
Standarddeutsche. Spätestens nach dem 1. Weltkrieg verfestigt sich nach und nach der Sprachname Luxemburgisch/Lëtzebuergesch oder auch einfach Eis Sprooch/Ons Sprooch, auch wenn damit immer noch ein Dialekt des Deutschen gemeint war.

Der Dialekt wurden von allen gesellschaftlichen Schichten gleichermaßen verwendet, doch blieb er ausschließlich auf jegliche nähesprachlichen Situationen der Mündlichkeit beschränkt. Alle formalen und öffentlichen, sowie praktisch alle schriftlichen Domänen waren uneingeschränkt dem Deutschen und Französischen vorbehalten (z.B. in der Abgeordnetenkammer, öffentlichen Ansprachen, Geburts- und Todesanzeigen). Aus diesem Grund kann ein weitgehend negatives Prestige für den Dialekt angenommen werden.

Die zweite Hälfte des 19. Jh. erlebte darüber hinaus auch eine Ausweitung der deutschen Schriftsprache nicht nur als Schulfach und Schulsprache, sondern auch als Verwaltungssprache, indem u.a. mehr öffentliche Aushänge auf Deutsch oder zweisprachig auf Deutsch und Französisch publiziert wurden (vgl. Beyer et al. 2014). Dennoch blieb das Französische (bis heute) als wichtigste Amtssprache bestehen. Diese stärkere Implementierung des Standarddeutschen trug zunächst jedoch auch zu einer weiteren Verfestigung des Überdachungsverhältnisses bei, in dem der moselfränkische Dialekt als die Varietät der Sprechsprachlichkeit fungiert und das Standarddeutsche als die 'linguistisch zugeordnete Kultursprache' (Kloss 1952: 20).

Doch unterlag diese Überdachung durch die traumatischen Erfahrungen der zwei Weltkriege in Luxemburg einem tiefgreifenden Wandel. Nicht zuletzt ausgelöst und verstärkt durch die Verletzung der Neutralität Luxemburgs im Ersten Weltkrieg und durch die Besetzung und die Gewaltverbrechen durch das Deutsche Reich im Zweiten Weltkrieg kam es zu einem tiefgreifenden Wandel in den Spracheinstellungen zum Deutschen (verstanden sowohl als Standardsprache als auch als Varietätengefüge) und weiterhin zu Verschiebungen der Mehrsprachigkeit und der Überdachung. Neben der gezielten Verdrängung des Französischen aus Öffentlichkeit, Schule und Verwaltung instrumentalisierte die vom Deutschen Reich eingerichtete und gesteuerte Zivilverwaltung Luxemburgs den Dialektstatus des Moselfränkischen: Durch die angenommenen Überdachung durch das Standarddeutsche wurde argumentativ auch eine ethnische Zugehörigkeit zum Deutschen Reich insinuiert. Eine verkappte Volksbefragung im Oktober 1941 ('Personenstandsaufnahme') verfehlte jedoch ihr Ziel, da die luxemburgische Bevölkerung trotz massiver Einschüchterung ihren Dialekt mit großer Mehrheit als 'Luxemburgisch' bezeichnete, sodass die Ergebnisse der Personenstandsaufnahme niemals veröffentlicht wurden. Aus diesem Widerstand gegen das Deutsche Reich und gegen die deutsche Sprache als die 'Sprache der Besatzer' erwuchs in kurzer Zeit eine attitudinale Dissoziierung des Dialekts aus dem Varietätengefüge des Deutschen. Trotz klar erkennbarer historischer Ähnlichkeiten wurde mit dem Ende des Zweiten Weltkrieges der Dialekt als - zumindest in den Spracheinstellungen - unabhängig vom Deutschen angesehen, womit der Beginn der Auflösung des Überdachungsverhältnisses eingeleitet wurde. Bereits nach dem Ersten Weltkrieg nahmen anti-deutsche Ressentiments zu und diese manifestierten sich in einer Hinwendung zu Frankreich und dem Französischen, z.B. in amtlichem Schrifttum. Diese Tendenzen verstärkten sich entsprechend nach dem Zweiten Weltkrieg und trugen zur weitgehenden Diskreditierung des Deutschen in Luxemburg bei.

\section{5. Überdachung seit 1945}

Die zunächst radikale Abkehr von allem Deutschen in der Zeit der Neuordnung nach dem Zweiten Weltkrieg ließ sich jedoch nicht konsequent umsetzen, da die über Jahrhunderte 
gewachsene Verankerung des Deutschen besonders im Schulsystem und der mittleren bis unteren Verwaltungsebene zu manifest war. Doch wurde dem Deutschen bis in die 1980er Jahre hinein ein ausgesprochen negatives Prestige zugeschrieben, so dass es weit hinter dem Französischen und Luxemburgischen rangierte. Das Standarddeutsche behielt zwar den Großteil seiner angestammten Domänen, wurde jedoch aus allen nationalsymbolisch oder identifikatorischen Kontexten verdrängt: Seit dieser Zeit ist es undenkbar und unmöglich, Deutsch in der Abgeordnetenkammer, für öffentliche Ansprachen oder für Geburts- und Todesanzeigen zu verwenden. Seit jüngster Zeit verschieben sich die Prestigeverhältnisse erneut und das Deutsche ist wieder mit zunehmend positivem Prestige assoziiert, was sich u.a. darin niederschlägt, dass stärker aus dem Deutschen ins Luxemburgische entlehnt wird (vgl. Conrad 2017).

Die Phase seit 1945 ist im Wesentlichen durch zwei Tendenzen geprägt: Erstens entwickelt sich die etablierte Mehrsprachigkeit mit Deutsch, Französisch und Luxemburgisch zu einer komplexen sozialen Mehrsprachigkeit, wodurch infolge der starken Immigration (Ausländeranteil 2017: 47,7\%) und der Internationalisierung der Arbeitswelt nicht mehr jeder Einwohner über dieselben Sprachkompetenzen verfügt. Diese Asymmetrien manifestieren sich in der Schwierigkeit, eindeutige und stabile Angaben zum Sprachengebrauch in den meisten Domänen und Situationen zu machen. Viele Sprachenwahlen werden ad-hoc in Abhängigkeit von den überlappenden Sprachkompetenzen der Beteiligten ausgehandelt. Diese Verschiebung im Mehrsprachigkeitsgefüge trägt einerseits dazu bei, dass sich das Französische immer mehr zur omnipräsenten Umgangssprache in der Öffentlichkeit und an vielen Arbeitsplätzen ausbreitet. Dazu kommt noch die rapide zunehmende Präsenz des Englischen (Newton 2009). Andererseits nimmt aber auch die Mehrsprachigkeit an sich zu und damit auch die Notwendigkeit, mit einem mehrsprachigen Repertoire flexibel auf unterschiedlichste kommunikative Anforderungen reagieren zu können; vgl. ausführlicher die makrosoziolinguistischen Überblicksdarstellungen von Weber (1994), Horner/Weber (2008, 2016) und Fehlen (2009) sowie die mikrosoziolinguistischen Studien von Franziskus (2013) und Belling (2015).

Zweitens, und relevanter im vorliegenden Kontext, kommt es zu einem funktionalen und strukturellen Ausbau des Luxemburgischen (vgl. Kloss 1986, Gilles 1999). Die zunehmend positiven Spracheinstellungen gegenüber dem Luxemburgischen führen dazu, dass sich in dieser Phase der Sprachname Lëtzebuergesch verfestigt und dass damit indirekt auch das Wissen um die historische Einordnung in das Dialektkontinuum mit den verwandten moselfränkischen Dialekten in Deutschland nach und nach zurückgeht.

Die attitudinale Dissoziation vom Deutschen bedingte in der Nachkriegszeit eine verstärkte Besinnung auf die Erstsprache ('Mammesprooch'), die die Luxemburger am meisten verwenden und in allen Domänen und Situationen einsetzen (können). Konträr zur Einschätzung der gesellschaftlichen Eliten und auch der zeitgenössischen luxemburgischen Sprachwissenschaftler (vgl. z.B. Hoffmann (1974) oder ironisch Bruch (1954b: 36f): "Wir erhoben die von reichsdeutschen Sprachreinigern als Bastard geächtete Mundart in den Adelsstand einer Langue Luxembourgeoise") setzte sich in weiten Teilen der Bevölkerung die Überzeugung durch, dass es sich beim Luxemburgischen um eine eigenständige Sprache handele. Infolge der Lobbyarbeit verschiedener Akteure kam es 1984 zur Verabschiedung eines Sprachengesetzes. Besonders einflussreich war hier die Sprachgesellschaft Actioun Lëtzebuergesch, die Hilfestellungen für alltägliche Sprachaufgaben (Briefsteller, Namenslisten, Entwürfe für Familienanzeigen und Reden etc.) auf Luxemburgisch anbot (vgl. Fehlen 2016).

Das Sprachengesetz von 1984 ('Loi du 24 février 1984 sur le régime des langues') deklariert zum ersten Mal in der Geschichte Luxemburgs das Luxemburgische als 
Nationalsprache ('Art. 1er Langue nationale: La langue nationale des Luxembourgeois est le luxembourgeois.') und verdeutlicht damit den herausgehobenen Status des Luxemburgischen. Als Amtssprachen ('langues administratives') werden die drei Sprachen Französisch, Deutsch, Luxemburgisch festgelegt und damit wird zum ersten Mal die Dreisprachigkeit des Landes festgehalten. Da das Rechtssystem auf dem Code civil basiert, bleibt das Französische die alleinig gültige Rechtssprache. Trotz seiner Kürze und seiner fehlenden konkreten Ausbaumaßnahmen stellt dieses Sprachengesetz einen Meilenstein der sprachenpolitischen Entwicklung des Landes dar, indem der aktuelle Zustand des Luxemburgischen sowie der Mehrsprachigkeit rechtlich und symbolisch festgeschrieben werden. Für die in den nächsten Jahren anstehende Verfassungsreform ist perspektivisch vorgesehen, entsprechende Artikel in die luxemburgische Verfassung aufzunehmen, wodurch der rechtliche und nationalsymbolische Status des Luxemburgischen noch weiter verfestigt werden wird.

Inwieweit das Sprachengesetz tatsächlichen Einfluss auf die parallel einsetzenden Ausbauprozesse des Luxemburgischen hat, ist bis heute umstritten. Unstreitig ist, dass in dieser Phase das Luxemburgische einen zunehmenden funktionalen und strukturellen Ausbau erfährt. Aufgrund der unklaren bzw. staatlich kaum gestützten Sprachplanung und Sprachpolitik spricht Fehlen (2015) hier von einem 'ungeplanten Ausbau'.

Anzuführen ist zunächst die Zunahme des Luxemburgischen in der Schriftlichkeit. Seit den 1980er Jahren werden zunehmend größere literarische Texte, beginnend mit den Romanen von Guy Rewenig, Josy Braun oder Roger Manderscheid, auf Luxemburgisch verfasst. Diese Werke belegen, dass literarische Kreativität im Luxemburgischen durchaus möglich ist. Das literarische Feld hat sich seitdem kontinuierlich weiterentwickelt, sodass heute die Existenz einer luxemburgischen Literatursprache in allen Gattungen angenommen werden kann (vgl. Berg 2006). Dabei dürften Kinder- und Jugendbücher sowie Comics die wichtigsten Textsorten repräsentieren. Dazu kommen zahlreiche Übersetzungen auf Luxemburgisch (z.B. Harry Potter-Romane, Neues Testament, Comics). In der privaten Schriftlichkeit wurden zunächst Briefe, Notizen und Tagebücher auf Luxemburgisch verfasst. Mit der Verbreitung der digitalen Medien seit den 1990er Jahren erfuhr dieser Bereich einen immensen Zuwachs, dergestalt, dass heute praktisch alle E-Mails, SMS, WhatsAppNachrichten, Facebook-Einträge, Kommentare auf Webseiten etc. zwischen LuxemburgerInnen faktisch einsprachig luxemburgisch verfasst werden. Dies trifft auch auf emotional besetzte Textsorten wie Geburts- und Traueranzeigen zu, die vorher konsistent in Französisch oder Deutsch verfasst waren. Ausgehend von dieser Entwicklung ist auch eine konstante Zunahme, allerdings noch auf niedrigem Niveau, luxemburgischer Schriftlichkeit in öffentlichen Domänen zu verzeichnen, hier häufig in Kombination mit dem Französischen (Broschüren, Anzeigen, Reklame, Aufschriften, Beschilderung etc.). Dazu gehört auch die Ausarbeitung einer luxemburgischen Version einer 'Wikipedia' (http://lb.wikipedia.com), die zurzeit ca. 50.000 (mehr oder weniger ausführliche) Artikel enthält, die zum Ausbau des Wortschatzes und der Fachsprachlichkeit im Allgemeinen beitragen. Zwar dürften die Leserzahlen wie bei anderen Wikipedien, die in Klein- oder Minderheitensprachen verfasst sind, relativ gering sein, doch dienen sie der kleineren Gruppe von Mitarbeitern dazu, die Leistungsfähigkeit der Sprache aktiv zur fördern und zu entwickeln (vgl. dazu Gilles 2011 sowie Kramer 1984, Weber 1994 zum lexikalischen Ausbau).

Im Bereich der Mündlichkeit besetzt das Luxemburgische Domänen, die vormals ganz oder überwiegend dem Französischen und Deutschen vorbehalten waren (vgl. Berg 1993, Weber 1994). Die Redensprache in der Abgeordnetenkammer wie generell der gesamten internen wie externen politischen Diskussionen ist heute ausschließlich Luxemburgisch. Zudem werden die Parlamentsreden sämtlich transkribiert und veröffentlicht und 
repräsentieren ein umfangreiches schriftliches Textkorpus. Eine wichtige Rolle bei der 'Popularisierung' (vgl. Mattheier 1998) des Luxemburgischen spielen die katholische Kirche und die audiovisuellen Medien. Nach dem zweiten vatikanischen Konzil konnte das Luxemburgische vermehrt eingesetzt werden. Heute sind die meisten Predigten, teilweise auch die Lesung aus dem Evangelium auf Luxemburgisch, während die formalisierten liturgischen Teile des Gottesdiensts deutsch oder französisch sind. An der Universität Luxemburg werden bestimmte Veranstaltungen und ganze Studiengänge auf Luxemburgisch angeboten. Die meisten luxemburgischen Radio- und TV-Sender verwenden ausschließlich Luxemburgisch (während die geschriebene Presse nahezu ausschließlich auf Deutsch und Französisch arbeitet). Der älteste Sender, Radio Luxemburg (heute: RTL - 'Radio Télé Lëtzebuerg'), sendet seit der Gründung in den 1930er Jahren zunehmend auf Luxemburgisch und ist bis heute der meist gehörte und geschaute Medienanbieter (vgl. Hoffmann 1996: 113ff.). Aufgrund des großen Erfolgs entstanden weitere, kleinere Radiosender (z.B. eldoradio oder 100komma7). Befördert durch diese Medien entstehen seit ca. 30 Jahren vermehrt auch Kinofilme und TV-Serien auf Luxemburgisch.

In jüngster Zeit sind die audiovisuellen Medien dazu übergegangen, Mündlichkeit und Schriftlichkeit dahingehend zu vernetzen, dass die meisten Wortbeiträge in Textform auch auf begleitenden Internetplattformen oder Facebook publiziert werden. Diese Beiträge werden von den Lesern kommentiert, woraus sich eine spezifische schriftliche Interaktionsstruktur entwickelt hat. Aufgrund ihrer hohen Zuschauer- und Hörerzahlen sind diese Medien als einflussreiche Multiplikatoren für die Wortschatzentwicklung und die Implementierung und Verfestigung der Schriftlichkeit des Luxemburgischen anzusehen (Gilles 2015). Öffentliche Reden und Vorträge werden zunehmend ebenfalls auf Luxemburgisch gehalten, dazu gehören auch zentrale Staatsakte wie die jährliche Weihnachtsansprache des Großherzogs oder die Rede zur Lage der Nation des Staatsministers (seit 1995), die vordem dem Französischen vorbehalten waren.

Das Luxemburgische hat somit neue Domänen entwickelt, die durch den Rückgang des Deutschen, weniger des Französischen, frei wurden, oder die überhaupt erst neu infolge der Digitalisierung der Kommunikation entstanden sind (Online-Textsorten/-diskurse). Wie die Beispiele belegen, wird der Gebrauch prinzipiell weder durch den Faktor privat/öffentlich noch durch den Formalitätsgrad beschränkt. In der Diskussion des Kloss'schen Ausbaubegriffs durch Berg (1993: 106-114) befindet sich das Luxemburgische demnach auf dem Weg zu den höchsten Ausbaustufen, wodurch die Charakterisierung als Ausbausprache gerechtfertigt wird, i.e. "3. Phase: Ausbau der Sachprosa, Lehrbücher für Grundschulen, kleine Originalaufsätze im Bereich Heimatforschung, volkstümliche Zeitschriften, Predigten, Rundfunkvorträge". Zwar sieht Berg (1993) bereits damals Ansätze zur "4. Phase: Lehrbücher über alle möglichen Wissensgebiete, Originalforschungen zur Heimatkunde, anspruchsvolle Zeitschriften" und "5. Phase: Größere Originalforschungen auf allen Wissensgebieten, Verwendung in amtlichen Schriftstücken und im Wirtschaftsleben, ganze Zeitungen in der Sprache", doch hat sich dieser prognostizierte Ausbau bis heute (noch) nicht eingestellt. Berg (1993) differenziert entsprechend zwischen 'Ausbauwilligkeit' und 'Ausbaufähigkeit': Erstere ist durch die potenzielle Omnipräsenz des Luxemburgischen in allen Bereichen gegeben, doch wird die Ausbaufähigkeit, insbesondere in den erwähnten Phasen vier und fünf, bis heute stark eingeschränkt, da diese formalen Domänen in der mehrsprachigen Gesellschaft zurzeit fest durch andere Sprachen besetzt sind. Inwieweit das Luxemburgische hier also tatsächlich weiter ausgebaut werden kann, wird davon abhängen, ob Verschiebungen in der Mehrsprachigkeit weitere Freiräume für das Luxemburgische eröffnen, sei es durch 
Verdrängungen von angestammt deutschen oder französischen Domänen oder durch Koexistenz.

Obwohl in jüngster Zeit durchaus Zuwächse in konzeptionell-schriftlichen Domänen zu verzeichnen sind, so liegen die am stärksten genutzten Domänen des Luxemburgischen dennoch in konzeptionell-mündlichen Bereichen, die sowohl medial-mündlich wie medialschriftlich realisiert sein können. In Gilles (2011) wird diese Konstellation als 'konzeptionelle Diglossie' bezeichnet, die die mediale Diglossie des 19. Jh., in der das Luxemburgische nur medial-mündlich existierte, abgelöst hat. Für diese Verwendungsweisen des Luxemburgischen erscheint m.E. die Charakterisierung als 'Vernakularsprache' angebracht.

Während das Französische durchweg als Fremdsprache eingestuft (und in den Schule unterrichtet) wird, so sind die Verhältnisse für das Deutsche weniger eindeutig: Wie Wagner $(2015,2017)$ für den Sprachunterricht zeigt, oszillieren die Einschätzungen der Deutschlehrer zwischen Fremdsprache, Zweitsprache und Erstsprache - letztere aufgrund der Tatsache, dass das Deutsche als Alphabetisierungssprache und bis zu den mittleren Klassenstufen des Gymnasiums als wichtigste Schulsprache fungiert.

Zusammenfassend kann festgehalten werden, dass das Luxemburgische heute strukturell betrachtet nicht mehr vom Deutschen (und insbesondere nicht vom Französischen) überdacht wird (vgl. dazu auch Ammon 1994). Andererseits kann jedoch eine partielle funktionale Überdachung durch das Standarddeutsche und Französische angenommen werden, da diese Sprachen nach wie vor wichtige schriftliche Domänen besetzen.

\section{Standardisierung des Luxemburgischen}

Die Veränderungen der Überdachung sind begleitet von verschiedenen Standardisierungsakten; vgl. zur Standardisierungsgeschichte v.a. Newton (2002), Gilles/Moulin (2003), Moulin (2006) und Stell (2006). Mit der in Abschnitt 2 bereits beschriebenen Homogenisierung der regionalen Variation im Dialektausgleich ist eine Varietät des Luxemburgischen entstanden, die als die allgemein akzeptierte Varietät angesehen wird. Im Sinne von Haugens (1966) Standardisierungsmodell vollzieht sich in diesem Dialektausgleich die Phase der Selektion der zukünftigen Standardvarietät.

Bedingt durch die allgemeine Zunahme der luxemburgischen Schriftlichkeit sind zahlreiche Ansätze zur Standardisierung der Orthografie zu verzeichnen; vgl. den Überblick in Newton (2000). Während im 19. Jh. noch Individualschreibweisen verschiedener Autoren ohne Anspruch auf weitere Verbreitung vorherrschten, so entstand mit den ersten Schulbüchern die Notwendigkeit einer einheitlichen Schreibung. 1914 wurde im Anhang eines erfolgreichen Schulbuchs die erste quasi-offizielle Orthografie vorgestellt (Welter 1914), die zur einfacheren Lesbarkeit an das Deutsche angelehnt war (z.B. $\langle i e\rangle /[i:],\langle v\rangle$ neben $\langle\mathrm{f}\rangle,\langle\mathrm{z}\rangle$ neben $\langle\mathrm{tz}\rangle,<\mathrm{e}\rangle$ und $<$ ä $>$ für [æ]; Groß-/Kleinschreibung), jedoch insbesondere im Vokalismus eigene graphematische Lösungen etablierte (<â>/[a:], <ê $\left.>/[\mathrm{e}:],<e ̈>/[ə],<\mathrm{e}^{\prime}>/[3 \mathrm{r}],<0^{\prime}>/[\partial \mho]\right)$. Obwohl nie staatlich offiziell sanktioniert, fand diese so genannte 'Welter-EngelmannOrthografie' weite Akzeptanz über die Schule hinaus und war bis weit nach dem 2. Weltkrieg in Gebrauch. Nach dem zweiten Weltkrieg wurde eine neue Orthografie entwickelt, in der sämtliche Anleihen an das Deutsche eliminiert wurden (Arrêté 1946). In dieser 'Lezebuurjer Ortografi' (auch 'Margue-Feltes-Orthografie') wurde ein weitgehend phonetisch orientiertes

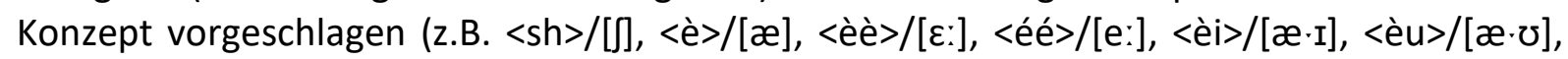
Abschaffung von $\langle\mathrm{v}\rangle /[\mathrm{f}],\langle\beta\rangle,\langle q>$, Kleinschreibung, phonetische Integration aller französischen Fremd- und Lehnwörter). Aufgrund des radikal neuen Schriftbildes (shtèet 
'steht', ssalii 'Salut', filoloji 'Philologie', ègzaamen 'Examen'), das zudem viele Diakritika enthielt, um alle vokalischen Differenzierungen ausdrücken zu können, fand die neue Orthografie in der Öffentlichkeit keine Akzeptanz und sie wurde bereits kurz nach der Einführung wieder zurückgenommen. Im Zuge der Ausarbeitung des Luxemburger Wörterbuches (LWB) (1950-1977) wurde 1975 eine reformierte Orthografie vorgestellt, die wiederum mehr Anleihen am gewohnten deutschen Schriftbild zuließ (Arrêté 1975). Leicht reformiert 1999 (Reglèment 1999), nimmt ihre Implementierung bis heute stetig zu (vgl. Gilles 2015). Die wachsende Akzeptanz der orthografischen Normen trug auch dazu bei, dass 2006 aus einer privaten Initiative das erste Korrekturprogramm entstand (http://spellchecker.lu), das für viele LuxemburgerInnen eine häufig konsultierte Hilfe für das korrekte Schreiben darstellt.

Im Bereich der lexikografischen Erschließung begann eine substanzielle Standardisierung erst in den 2000er Jahren. Im 19. Jh. und 20. Jh. entstanden zwar eine ganze Reihe Wörterbücher, die jedoch immer explizit der Dokumentation des Dialekts dienten (vgl. Lexikon der Luxemburger Umgangssprache (LLU) (1847), Wörterbuch der luxemburgischen Mundart (WLM) (1906), Luxemburger Wörterbuch (LWB) (1950-1977)). Erst mit dem Entstehen des Lëtzebuerger Online Dictionnaires (LOD) seit 2007 kann eine stärker präskriptive Lexikografie angenommen werden (vgl. Ecker 2013). Im LOD werden zum ersten Mal nicht nur die aktuellen orthografischen Normen für den gesamten Wortschatz angewandt, sondern auch bewusste Entscheidungen getroffen, welche Lexeme zum Wortschatz gehören und welche nicht. Der intensive Sprachkontakt mit dem Französischen und Deutschen bedingt zahlreiche Diskussionen, ob Lehn- oder Fremdwort aus dem Deutschen oder dem Französischen präferiert werden sollten (vgl. Conrad 2017).

Ähnlich verhält es sich mit der Grammatikschreibung: Umfangreiche Grammatiken, die ansatzweise auch wissenschaftlichen Ansprüchen genügen und die über den rein praktischen Bedarf etwa im Sprachunterricht hinausgehen, entstehen erst seit dem Beginn des 21. Jh. (Schanen/Zimmer 2012). Während der muttersprachliche Unterricht in den Schulen bis heute als kaum existent bezeichnet werden kann, so ist ein konstanter Ausbau im Bereich 'Luxemburgisch als Fremdsprache' beobachtbar (Weber-Messerich 2011), verbunden mit der Entwicklung von zahlreichen Lehrbüchern und Lehrmaterialien.

Zusammenfassend ergibt sich damit für die Standardisierung, dass die Phasen der Selektion und Kodifizierung als abgeschlossen betrachtet werden können. Trotz der zögerlichen sprachplanerischen Einstellung des Staats, sind Ansätze zur Implementierung der Normen und ihrer funktionalen Elaboration zwar deutlich erkennbar, doch kann noch nicht von einer weiteren gesellschaftlichen Durchsetzung gesprochen werden, insbesondere da das Luxemburgische bis heute noch nicht einmal ansatzweise als Schulfach und Schulsprache institutionalisiert ist. Insgesamt kann die Standardisierung heute somit auf mittlerem Niveau lokalisiert werden.

\section{Resümee}

Die luxemburgische Sprach(en)geschichte bietet ein eindrückliches Beispiel für den Umbau von Überdachungsverhältnissen infolge von nationalstaatlichen Entwicklungen und der damit verbundenen Genese einer neuen Nationalsprache. Als zentrale Umbruchsphase für Luxemburg wurde der 2. Weltkrieg identifiziert. In dieser Zeit wurde die vorherige strukturelle und funktionale Überdachung der moselfränkischen Dialekte durch das Deutsche aufgelöst und ersetzt durch ein neues Varietätensystem, dass sich zwischen den angestammten 
Dialekten und der emergenten luxemburgischen Standardvarietät aufspannt. Die Standardisierung und der Dialektausgleichsprozess im Verlauf des 20. Jh. führten zur Verringerung der strukturellen (vertikalen) Distanz zwischen den angestammten Dialekten und der emergenten luxemburgischen Standardvarietät. Dabei übernimmt die luxemburgische Standardvarietät (bislang) nur in eingeschränktem Maße ihre standardsprachlichen Funktionen, die überwiegend noch durch das Deutsche und Französische besetzt sind und möglicherweise auch bleiben. Daraus folgt, dass im aktuellen Varietätensystem weiterhin eine partielle funktionale Überdachung durch diese beiden Sprachen angenommen werden muss, die sich nicht nur in der Dominanz in den konzeptionellschriftlichen Domänen, sondern auch in vielfältigen Entlehnungen in das Luxemburgische manifestiert. Im Bereich der medialen und konzeptionellen Mündlichkeit hat sich eine teilstandardisierte, hinsichtlich Wortschatz hoch-variable Vernakularsprache etabliert. Die folgende Abbildung fasst die beschriebenen Prozesse zusammen.

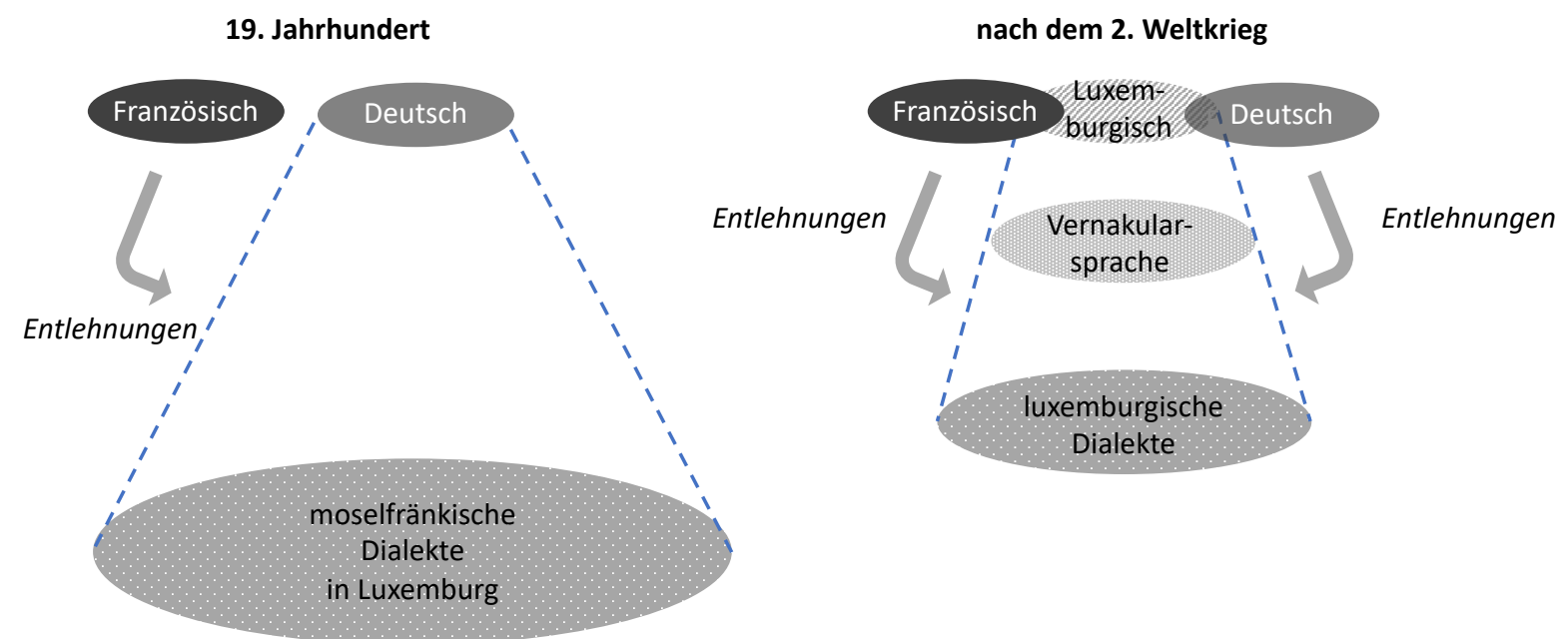

Abbildung 3 Gegenüberstellung der Überdachungsverhältnisse in Luxemburg vor und nach dem 2. Weltkrieg

Während etwa in Deutschland der moselfränkische Dialekt durch den zunehmenden Druck der Standardsprache in einem diaglossischen Verhältnis nivelliert wird, so führte die staatliche Eigenständigkeit sowie die fest etablierte Mehrsprachigkeit in Luxemburg zu einer gänzlich anderen Entwicklung. Sowohl im Sprachgebrauch als auch in der Sprachstruktur hat sich damit zwischen Deutschland und Luxemburg eine Sprachgrenze herausgebildet. Die folgende Übersicht fasst die wichtigsten distinktiven Merkmale beider Varietäten zusammen:

\begin{tabular}{|l|l|}
\hline Moselfränkisch in Deutschland & Luxemburgisch \\
\hline $\begin{array}{l}\text { Dialekt; Abbau- und Ausgleichsprozesse in } \\
\text { Richtung auf Regionalsprache des } \\
\text { Deutschen }\end{array}$ & $\begin{array}{l}\text { Vernakularsprache; Dialektausgleich } \\
\text { innerhalb Luxemburgs }\end{array}$ \\
\hline $\begin{array}{l}\text { Standarddeutsch überdacht das } \\
\text { Dialektkontinuum }\end{array}$ & $\begin{array}{l}\text { neu entwickelte Überdachung mit eigener } \\
\text { Standardisierung }\end{array}$ \\
\hline starke situative Einschränkungen & kaum situative Einschränkungen \\
\hline niedriges offenes, eher verdecktes Prestige & hohes offenes Prestige \\
\hline intergenerationelle Weitergabe gefährdet & intergenerationelle Weitergabe intakt \\
\hline
\end{tabular}


keine Schriftlichkeit

entstehende Schriftlichkeit, im

konzeptionell-mündlichen Bereich fest

etabliert

\section{Literatur}

Ammon, Ulrich. 1994. Was ist ein deutscher Dialekt? Präzisierungsversuch einer Grundfrage der deutschen Dialektologie und der in ihr enthaltenen ungelösten Forschungsaufgaben. In Klaus Mattheier \& Peter Wiesinger (eds.), Dialektologie des Deutschen, 369-384. Niemeyer.

Arrêté 1946 = Arrêté ministériel du 5 juin 1946 portant fixation d'un système d'orthographe luxembourgeoise. Mémorial du Grand-Duché de Luxembourg 40. 637-641.

Arrêté 1975 = Arrêté ministériel du 10 octobre 1975 portant réforme d'un système officiel d'orthographe luxembourgeoise. Mémorial - Journal Officiel du Grand-Duché de Luxembourg / Amtsblatt des Großgherzogtums Luxemburg (B68). 1365-1390.

Bach, Adolf. 1933. Die sprachliche Stellung Luxemburgs. Jahrbuch der Luxemburgischen Sprachgesellschaft 1931/1932 32. 3-13, 4. Ktn.

Beckers, Hartmut. 1980. Westmitteldeutsch. In Hans Pete Althaus, Helmut Henne \& Herbert Ernst Wiegand (eds.), Lexikon der germanistischen Linguistik, 468-473. 2. Auflage. Tübingen.

Belling, Luc. 2015. Mediale und sprachliche Möglichkeitsräume in digitaler Schriftlichkeit - Eine Studie zu Facebook-Pinnwänden in Luxemburg. Luxembourg: University of Luxembourg PhD Dissertation.

Berg, Guy. 1993. "Mir wëlle bleiwe, wat mir sin". Soziolinguistische und sprachtypologische Betrachtungen zur luxemburgischen Mehrsprachigkeit. (Reihe Germanistische Linguistik 140). Tübingen: Niemeyer.

Berg, Guy. 2006. Abschied vom Dialekt. Zur lëtzebuergeschen belletristischen Gegenwartsliteratur. In Claudine Moulin \& Damaris Nübling (eds.), Perspektiven einer linguistischen Luxemburgistik. Studien zu Diachronie und Synchronie, 341-356. (Germanistische Bibliothek 25). Heidelberg: Winter.

Beyer, Rahel, Peter Gilles, Olivier Moliner \& Evelyn Ziegler. 2014. Sprachstandardisierung unter Mehrsprachigkeitsbedingungen: Das Deutsche in Luxemburg im 19. Jahrhundert. Jahrbuch für germanistische Sprachgeschichte, 283-298. Berlin; New York: de Gruyter.

Bourg, J. P. 1895. Die Luxemburger Mundart. Ons Hémecht 1. 205-207, 229-231, 258-262, 287-291, 315-318, 351-355; (1896): S. 40-42, 68-72, 127-129, 172-175, 210-213, 228-231, 275-278, 300-303, 332-335, 361-363, 403-407, 423-425.

Bruch, Robert. 1949. La vélarisation ripuaire des occlusives dentales. Revue Trisemestrielle 8. 78-90.

Bruch, Robert. 1952. Die Mundart des Nordöslings. Jahrbuch der luxemburgischen Sprachgesellschaft. 1-50.

Bruch, Robert. 1953. Grundlegung einer Geschichte des Luxemburgischen. (Publications Littéraires et Scientifiques Du Ministère de l'éducation Nationale). Luxemburg: P. Linden, Hofbuchdrucker.

Bruch, Robert. 1954a. Das Luxemburgische im Westfränkischen Kreis. (Publications Littéraires et Scientifiques Du Ministère de l'Éducation Nationale 2). Luxemburg: Linden.

Bruch, Robert. 1954b. Deutsche Spracherziehung - oder Sprachunterricht für Luxemburger...? Journal des Professeurs (Luxemburg), 30-55. (41). Luxemburg. 
Bruch, Robert. 1955. Précis populaire de grammaire Luxembourgeois. . Vol. 4/6. Luxembourg: Linden.

Bruch, Robert. 1963. Germanische und romanische ê- und ô-Diphthongierungen. In Ludwig Erich Schmitt (ed.), Deutsche Wortforschung in europäischen Bezügen. Untersuchungen zum Deutschen Wortatlas, vol. 2, 409-467. Gießen: Schmitz.

Conrad, François. 2017. Variation durch Sprachkontakt. (Luxemburg-Studien / Études Luxembourgeoises 14). Frankfurt / New York: Peter Lang.

Darquennes, Jeroen. Art. 41. Komplexe Überdachung III: Belgien. in diesem Band

Ecker, Alexandre. 2013. lod.lu: dictionnaire ou dictionnaires? Lexique( 21). 101-115.

Engelmann, René. 1910. Der Vokalismus der Viandener Mundart. (Programm Grossherzogliches Gymasium Zu Diekirch Herausgegeben Am Schlusse Des Schuljahres 1909-1910). Diekirch: J. Schroell.

Fehlen, Fernand \& Andreas Heinz. 2016. Die Luxemburger Mehrsprachigkeit. Ergebnisse einer Volkszählung. Bielefeld: Transcript.

Fehlen, Fernand. 2009. BaleineBis: une enquête sur un marché linguistique multilingue en profonde mutation. Luxemburgs Sprachenmarkt im Wandel. Luxembourg: SESOPI Centre intercommaunitaire.

Fehlen, Fernand. 2014. Die Stellung des Französischen in Luxemburg. Von der Prestigesprache zur Verkehrssprache. In Heinz Sieburg (ed.), Vielfalt der Sprachen - Varianz der Perspektiven, 37-80. Bielefeld: Transcript Verlag.

Fehlen, Fernand. 2015. Der ungeplante Ausbau des Luxemburgischen im Spannungsfeld von Germania und Romania. Quo vadis, Romania? Themenheft: „Entstehung von Sprachen" (45). 65-80.

Fehlen, Fernand. 2016. Nationalsprache und nationale Identität. Die Debatten im Vorfeld des Sprachengesetzes (1974-1984). In Norbert Franz, Thorsten Fuchshuber, Sonja Kmec, Jean-Paul Lehners \& Renée Wagener (eds.), Identitätsbildung und Partizipation im 19. und 20. Jahrhundert: Luxemburg im europäischen Kontext. (Études luxembourgeoises / Luxemburg-Studien Band 12). Frankfurt am Main: Peter Lang Edition.

Fehlen, Fernand. 2018. Die Grundlegung des Luxemburger multilingualen Habitus Die Einführung der zweisprachigen Primärschule 1843 und die Folgen. In M. Gardin \& Thomas Lenz (eds.), Die Schule der Nation. Bildungsgeschichte und Identität in Luxemburg, 55-75. Weinheim: Beltz.

Fleischer, Jürg \& Oliver Schallert. 2011. Historische Syntax des Deutschen: Eine Einführung. Tübingen: Gunter Narr Verlag.

Fontaine, Ed(mond) [= Dicks] de la. 1855. Versuch über die Orthographie der luxemburger deutschen Mundart. Luxemburg: V. Bück.

Franziskus, Anne. 2013. Getting by in a multilingual workplace: The language practices, ideologies and norms of cross-border workers in Luxembourg. PhD Dissertation. Luxembourg: University of Luxembourg.

Gilles, Peter. 1998. Die Emanzipation des Lëtzebuergeschen aus dem Gefüge der deutschen Mundarten. Zeitschrift für deutsche Philologie 117, Sonderheft. 20-35.

Gilles, Peter. 1999. Dialektausgleich im Lëtzebuergeschen. Zur phonetisch-phonologischen Fokussierung einer Nationalsprache. (Phonai 44). Tübingen: Niemeyer.

Gilles, Peter. 2000. Die Konstruktion einer Standardsprache. Zur Koinédebatte in der luxemburgischen Linguistik. In Dieter Stellmacher (ed.), Dialektologie zwischen Tradition und Neuansätzen. Beiträge der Internationalen Dialektologentagung. Göttingen, 19.-21. Oktober 1998, 200-212. (Zeitschrift Für Dialektologie Und Linguistik Beiheft 109). Stuttgart: Steiner. 
Gilles, Peter. 2006. Dialektausgleich im Luxemburgischen. In Claudine Moulin \& Damaris Nübling (eds.), Perspektiven einer linguistischen Luxemburgistik. Studien zu Diachronie und Synchronie, 1-27. (Germanistische Bibliothek 25). Heidelberg: Winter.

Gilles, Peter. 2009. Luxemburgisch: Vom Dialekt zur Nationalsprache. In Patrick Bousch, Tobias Chilla \& Christian Schulz (eds.), Der Luxemburg Atlas, 192-193. Köln: Emons.

Gilles, Peter. 2011. Mündlichkeit und Schriftlichkeit in der luxemburgischen Sprachgemeinschaft. In Georg Mein \& Heinz Sieburg (eds.), Medien des Wissens. Interdisziplinäre Aspekte von Medialität, 43-64. Bielefeld: transcript.

Gilles, Peter. 2011. Mündlichkeit und Schriftlichkeit in der luxemburgischen Sprachgemeinschaft. In Georg Mein \& Heinz Sieburg (eds.), Medien des Wissens. Interdisziplinäre Aspekte von Medialität, 43-64. Bielefeld: transcript.

Gilles, Peter. 2015. From status to corpus: Codification and implementation of spelling norms in Luxembourgish. In Wini Davies \& Evelyn Ziegler (eds.), Macro and micro language planning, 128-149. London: Palgrave Macmillan.

Gilles, Peter. im Druck. Luxembourgish. In Hans C. Boas, Ana Deumert, Marc Louden \& Péter Maitz (eds.), Varieties of German Worldwide. Oxford: Oxford University Press.

Gilles, Peter \& Claudine Moulin. 2003. Luxembourgish. In Ana Deumert \& Wim Vandenbussche (eds.), Germanic Standardizations. Past to Present, 303-329. (Impact 18). Amsterdam - Philadelphia: Benjamins.

Gilles, Peter \& Claudine Moulin. 2008. Der digitale luxemburgische Sprachatlas (LuxSA). Stand und Perspektiven. In Stephan Elspaß \& Werner König (eds.), Sprachgeographie digital - Die neue Generation der Sprachatlanten, 133-148. (Germanistische Linguistik 190191). Hildesheim/Zürich/New York: Olms.

Goossens, Jan. 1977. Deutsche Dialektologie. Berlin ; New York: De Gruyter.

Hardt, Mathias. 1843. Vocalismus der Sauer-mundart. Trier.

Haugen, Einar. 1966. Linguistics and language planning. In William Bright (ed.), Sociolinguistics. Proceedings of the UCLA sociolinguistics conference, 1964, 50-71. The Hague, Paris: Mouton.

Herrgen, Joachim \& Jürgen Erich Schmidt. 1989. Dialektalitätsareale und Dialektabbau. In Wolfgang Putschke (ed.), Dialektgeographie und Dialektologie. Günter Bellmann zum 60. Geburtstag von seinen Schülern und Freunden, 304-346, 5 Ktn. (DDG 90). Marburg: Elwert.

Hoffmann, Fernand. 1974. Standort Luxemburg. Luxemburg: Sankt-Paulus-Druckerei.

Hoffmann, Fernand. 1996. Lëtzebuergesch, Spoken and Written, Developments and Desirabilities. In Gerald Newton (ed.), Luxembourg and Lëtzebuergesch: language and communication at the crossroads of Europe, 109-121. Oxford: Oxford UP.

Horner, Kristine \& Jean-Jacques Weber. 2008. The language situation in Luxembourg. Current Issues in Language Planning 9(1). 69-128.

Horner, Kristine \& Jean-Jacques Weber. 2016. The language situation in Luxembourg. In Robert B. Kaplan, Richard B. Baldauf Jr \& Nkonko Kamwangamalu (eds.), Language Planning in Europe: Cyprus, Iceland and Luxembourg, 170-230. Routledge.

Huss, Richard. 1927. Studien zum luxemburgischen Sprachatlas. (Beiträge Zur Luxemburgischen Sprach- Und Volkskunde 2). Luxemburg.

Keller, Rudolf Ernst. 1961. Luxemburgish. German Dialects. Phonology and Morphology. With Selected Texts, 248-297.

Klein, Peter. 1855. Die Sprache der Luxemburger. Besonderer abdruck aus dem zehnten jahresheft des vereins für väterl. geschichts- und alterthumskunde. Luxemburg. 
Kloss, Heinz. 1940. Vielsprachiges Land. Vom Nebeneinander und Durcheinander der Sprachen in Luxemburg. Deutschtum im Ausland 23(3/4). 62-65.

Kloss, Heinz. 1952. Die Entwicklung neuer Germanischer Kultursprachen. Von 1800 bis 1950. 1. Auflage. Vol. I. München: Pohl \& Co Verlagsbuchhandlung.

Kloss, Heinz. 1986. Der Stand der in Luxemburg gesprochenen Sprachen beim Jahresende 1984. Germanistische Mitteilungen: Zeitschrift fur Deutsche Sprache, Literatur und Kultur in Wissenschaft und Praxis 24. 83-94.

Koenig, Lucien. 1928. Auf dem Wege zu einer Grammatik der Luxemburger Mundart. Ein Beitrag zur Geschichte der Lux. Dialektliteratur. I. Teil. Luxemburg.

Kramer, Johannes. 1984. Zweisprachigkeit in den Benelux-Ländern. Hamburg: Buske.

Krier, Fernande. 2015. Der Präteritumsschwund im Luxemburgischen. Zeitschrift für Dialektologie und Linguistik 82(1). 47-82.

$\mathrm{LLU}=$ Gangler, Jean François. 1847. Lexicon der Luxemburger Umgangssprache (wie sie in und um Luxemburg gesprochen wird) mit hochdeutscher und französischer Uebersetzung und Erklärung. Luxemburg. Online unter: http://engelmann.uni.lu:8080/portal/wbb/woerterbuecher/woerterbuecher/llu/wbg ui.

LSA = Schmitt, Ludwig Erich [Hrsg.] (ed.). 1963. Luxemburgischer Sprachatlas. Laut- und Formenatlas von Robert Bruch. Für den Druck vorbereitet von Jan Goossens. (Deutscher Sprachatlas. Regionale Sprachatlanten 2). Marburg.

LWB = Luxemburgische Wörterbuchkommission (ed.). 1950. Luxemburger Wörterbuch. Im Auftrage der Großherzoglich Luxemburgischen Regierung herausgegeben von der Wörterbuchkommission, auf Grund der Sammlungen, die seit 1925 von der Luxemburgischen Sprachgesellschaft und seit 1935 von der Sprachwissenschaftlichen Sektion des Großherzoglichen Instituts veranstaltet worden sind. 4 Bände, 1 Nachtragsband. Luxemburg: Linden. Online unter: http://engelmann.uni.lu:8080/portal/WBB2009/LWB/wbgui_py?mainmode=\&lemid= \&prefix $=a \&$ mode $=\&$ open $w b=1$.

Mattheier, Klaus J. 1998. Allgemeine Aspekte einer Theorie des Sprachwandels. Besch, Werner / Betten, Anne / Reichmann, Oskar / Sonderegger, Stefan (Hg., 1998), Sprachgeschichte. Ein Handbuch zur Geschichte der deutschen Sprache und ihrer Erforschung. Berlin/New York: de Gruyter. (Handbücher zur Sprach- und Kommunikationswissenschaft 2.1). 824-836.

Meyer, Anton. 1829. E' Schrek ob de' Lezeburger Parnassus. Luxemburg: J. Lamort.

Meyer, Anton. 1845. Luxemburgische Gedichte und Fabeln, nebst einer grammatischen Einleitung und einer Wörtererklärung der dem Dialekt mehr oder weniger eigenartigen Ausdrücke von Gloden. Brüssel: Delevingne und Callewaert.

Ministère de la Culture (ed.). 2007. Lëtzebuerger Online Dictionnaire (LOD). Luxembourg. http://lod.lu.

Moliner, Olivier \& Evelyn Ziegler. 2017. Sprachenpolitik, Sprachenideologie und Sprachgebrauch. Das Deutsche in Luxemburg im 19. Jahrhundert (1795-1920). Sociolinguistica 31(1). 125-146.

Moulin, Claudine \& Michel Pauly (eds.). 2007. Die Rechnungsbücher der Stadt Luxemburg. Erstes Heft 1388 - 1399. Luxemburg: Stadtarchiv.

Moulin, Claudine. 2006. Grammatisierung und Standardisierung des Luxemburgischen. Eine grammatikographisch-sprachhistorische Annäherung. In Claudine Moulin \& Damaris Nübling (eds.), Perspektiven einer linguistischen Luxemburgistik. Studien zu Diachronie und Synchronie, 305-339. (Germanistische Bibliothek 25). Heidelberg: Winter. 
Neises, Diane. 2013. Levelling toward a Higher Standard? A Study on Dialect Perception and Its Potential Implications for Language Change in Luxembourg. York: University of York Master thesis.

Newton, Gerald. 1990. Central Franconian. In Charles V. J. Russ (ed.), The Dialects of Modern German. A Linguistic Survey, 136-209. London: Routledge.

Newton, Gerald. 1993. Allophonic variation in Luxemburgish palatal and alveolar-palatal fricatives: Discussion of an areal survey taken in 1979. In John L. Flood, Paul Salmon, Olive Syce \& Christopher Wells (eds.), "Das unsichtbare Band der Sprache". Studies in German Language and Linguistic History in Memory of Leslie Seiffert, 627-656. Stuttgart: Hans-Dieter Heinz - Akademischer Verlag.

Newton, Gerald. 1996b. Lëtzebuergesch and the establishment of national identity. In Gerald Newton (ed.), Luxembourg and Lëtzebuergesch. Language and communication at the crossroads of Europe, 181-216. Oxford [u.a.]: Clarendon Press.

Newton, Gerald. 2000. The spelling of Luxembourgish : Systems and developments since 1824. In Gerald Newton (ed.), Essays on politics, language and society in Luxembourg, 135162. Lewiston: Edwin Mellon Press.

Newton, Gerald. 2002. The Standardization of Luxembourgish. In Andrew A. Linn \& Nicola McLelland (eds.), Standardization. Studies from the Germanic Languages, 178-190. Amsterdam - Philadelphia: Benjamins.

Newton, Gerald. 2009. The English influence on Luxembourgish. In Falco Pfalzgraf (ed.), Englischer Sprachkontakt in den Varietäten des Deutschen $=$ English in contact with varieties of German, vol. 12, 185-214. (Österreichisches Deutsch, Sprache Der Gegenwart). Frankfurt a. M.: Peter Lang.

Newton, Gerald (ed.). 1996a. Luxembourg and Lëtzebuergesch. Language and communication at the crossroads of Europe. Oxford: Clarendon Press.

Palgen, Helene. 1931. Kurze Lautlehre der Mundart von Echternach. (Beiträge Zur Luxemburgischen Sprach- Und Volkskunde 4). Luxemburg: Linden \& Hansen.

Palgen, Helene. 1948. Studien zur Lautgeographie Luxemburgs. (Beiträge Zur Luxemburgischen Sprach- Und Volkskunde 6). Luxemburg.

Palgen, Helene. 1954. Vokalismus der Mundart von Knaphoscheid (Kanton Wilz). Jahrbuch der luxemburgischen Sprachgesellschaft. 3-18.

Pauly, Michel. 2013. Geschichte Luxemburgs. 2., überarbeitete Auflage. (C. H.Beck Wissen). München: Beck.

Ravida, Fausto. 2012. Graphematisch-phonologische Analyse der Luxemburger Rechnungsbücher (1388-1500). Ein Beitrag zur historischen Stadtprachenforschung. 1st ed. Heidelberg: Universitätsverlag Winter.

Reglèment 1999 = Orthographe Luxembourgeoise. Reglèment grand-ducal du 30 juillet 1999 portant réforme du système officiel d' orthographe luxembourgeoise. Mémorial / Amtsblatt des Grossherzogtums Luxemburg(A112). 2040-2048.

Ries, Ries. 1911. Essai d'une psychologie du peuple luxembourgeois. Diekirch: J. Schroell.

Rinnen, Henri. 1981. En éischten Zeien. Eis Sprooch. 11-15.

Schanen, François \& Jacqui Zimmer. 2012. Lëtzebuergesch Grammaire. Luxembourg: Éditions Schortgen.

Schiltz, Guillaume. 1997. A dialectometric analysis of the dialects in the Grand-Duchy of Luxemburg. In Alan R. Thomas (ed.), Issues and Methods in Dialectology, 94-108. Bangor: Central Print Unit.

Schmidt, Jürgen Erich \& Joachim Herrgen. 2011. Sprachdynamik: Eine Einführung in die moderne Regionalsprachenforschung. Berlin: Erich Schmidt. 
Schmidt, Jürgen Erich \& Robert Möller. Art. 16. Historisches Westdeutsch/Rheinisch (Moselfränkisch, Ripuarisch, Südniederfränkisch). in diesem Band.

Schützeichel, Rudolf. 1976. Die Grundlagen des westlichen Mitteldeutsche. Studien zur historischen Sprachgeographie. Tübingen.

Sinner, Carsten. 2013. Varietätenlinguistik: Eine Einführung. Tübingen: Narr Francke Attempto.

Solms, Hans-Joachim \& Jean-Paul Hoffmann. 1995. Letzebuergisch: von deutschsprachiger Mundart zur "historischen Einzelsprache / Nationalsprache". Vorüberlegungen zur Erforschung der luxemburgischen Koiné im 19. Jahrhundert. Bulletin linguistique, Ethnologique et Toponymique 26. 7-35.

Solms, Hans-Joachim \& Klaus-Peter Wegera. 1999. Luxemburger Druckersprache des 17. Jahrhunderts. Luxemburg: Imprimerie Centrale.

Stell, Gerald. 2006. Luxembourgish standardization: context, ideology and comparability with the case of West Frisian. Peeters Publishers.

Sturm, Hansjörg. 1988. Die Entwicklung des Vokalsystems im Luxemburgischen. Freiburg: Universität Freiburg Zulassungsarbeit im Fach Germanistik zur 1. wissenschaftlichen Staatsexamensprüfung.

Uhrmacher, Martin. 2009. Von der Grafschaft zum Großherzogtum. In Patrick Bousch und Tobias Chilla, Patrick Bousch, Christian Schulz \& Christophe Sohn (eds.), Der Luxemburg Atlas - Atlas du Luxembourg, 8-11. Köln: Emons.

Veldenz, Bruder Herrmann von. 2009. Leben der Gräfin Yolanda von Vianden. (Ed.) Claudine Moulin. (Beiträge zur luxemburgischen Sprach- und Volkskunde; Nr. 36. Sonderforschungsreihe Language and culture in medieval Luxembourg Bd. 5). Luxembourg: Institut Grand-Ducal, Section de linguistique, d'ethnologie et d'onomastique.

Wagner, Melanie. 2015. Germany in Secondary Schools in Luxembourg: the Implementation of Macro-Level Language Policies on the Micro Level of the Luxembourgish GermanLanguage Classroom. In Winifred V. Davies \& Evelyn Ziegler (eds.), Language Planning and Microlinguistics From Policy to Interaction and Vice Versa, 62-82. London: Palgrave Macmillan.

Wagner, Melanie. 2017. Luxemburg - ein Halbzentrum? Deutsch an Gymnasien in Luxemburg. Zeitschrift für deutsche Philologie(136). 145-167.

Weber-Messerich, Jacqueline. 2011. Luxemburgisch als Fremdsprache (LAF). In Peter Gilles \& Melanie Wagner (eds.), Linguistische und soziolinguistische Bausteine der Luxemburgistik, 337-345. (Mikroglottika. Minority Language Studies 4). Frankfurt a.M. [et al]: Peter Lang.

Weber, Nico. 1994. Sprachen und ihre Funktionen in Luxemburg. Zeitschrift für Dialektologie und Linguistik 66. 129-169.

Welter, Nikolaus. 1914. Das Luxemburgische und sein Schrifttum. 1. Auflage. Luxemburg: G. Soupert.

Wiesinger, Peter. 1983. Phonologische Vokalsysteme deutscher Dialekte. Ein synchronischer und diachronischer Überblick. In Werner Besch, Ulrich Knoop, Wolfgang Putschke \& Herbert Ernst Wiegand (eds.), Dialektologie. Ein Handbuch zur deutschen und allgemeinen Dialektforschung, vol. 2. Halbband, 830-899. Berlin/New York: de Gruyter.

WLM = 1906. Wörterbuch der luxemburgischen Mundart. Luxemburg. Online unter: http://engelmann.uni.lu:8080/portal/WBB2009/WLM/wbgui py? mainmode=\&lemid $=$ \&prefix $=a \&$ mode $=\& o p e n w b=1$. 